\title{
Artelogie
}

Recherche sur les arts, le patrimoine et la littérature de I'Amérique latine

$11 \mid 2017$

Délocalités, translocalités et activisme dans l'art électronique et biomédiale latino-américain

\section{Una excursión a las poéticas digitales latinoamericanas}

Anahí Alejandra Ré

\section{OpenEdition}

Journals

Edición electrónica

URL: http://journals.openedition.org/artelogie/1607

DOI: 10.4000/artelogie.1607

ISSN: 2115-6395

Editor

Association ESCAL

Referencia electrónica

Anahí Alejandra Ré, « Una excursión a las poéticas digitales latinoamericanas », Artelogie [En línea],

11 | 2017, Publicado el 28 diciembre 2017, consultado el 23 septiembre 2020. URL : http://

journals.openedition.org/artelogie/1607 ; DOI : https://doi.org/10.4000/artelogie.1607

Este documento fue generado automáticamente el 23 septiembre 2020

Association ESCAL 


\title{
Una excursión a las poéticas digitales latinoamericanas
}

\author{
Anahí Alejandra Ré
}

1 El libro Poesía y poéticas digitales. Electrónicas / tecnos / new-media en América latina: definiciones \& exploraciones, editado por Luis Correa-Díaz y Scott Weintraub, publicado en 2016 por la Universidad Central de Colombia, se presenta como una obra de referencia ineludible para quien se interese por el campo de los estudios literarios digitales, inscribiéndose en la tradición que abren ciertos trabajos "clásicos" en el área ${ }^{\mathrm{i}}$, aunque, a diferencia de los anteriores, esta se propone tornar la mirada hacia América Latina, y reúne para ello un conjunto de artículos de estudiosos especializados en sus corpus. Se constituye, así, como la primera en hacer foco en el resto del continente americano desde Estados Unidos, y también la primera de estas características que se edita en español (lengua materna de la mayoría de los autores compilados).

2 En su título, el libro apela a distintas denominaciones (electrónicas, tecnos, new-media), dejando en evidencia la inestabilidad de las conceptualizaciones categoriales: en el campo de la poesía y las poéticas digitales, múltiples definiciones coexisten y se usan de modos muy diversos. A veces, las que dicta el más espontáneo sentido común. Otras veces, las que problematizan seriamente los conceptos que se ponen en juego al intentar consolidar criterios. Precisamente, la introducción de este libro de referencia propone un gesto de orden y se aboca a analizar los distintos modos de concebir la poesía digital, problematizando usos del concepto, recabando antecedentes, haciendo un recorrido y adentrándose en profundidad en criterios que permitan establecer definiciones del género. Si bien, finalmente, opta por un concepto "sombrilla", es decir, "un concepto que determina un campo creativo y teórico-crítico que incluye obras y estudios sobre ellas que no responden bien a la imposición de una definición estricta", el trayecto conceptual que allí realiza Correa-Díaz resulta un valioso aporte a un estado de la cuestión para aquellos curiosos que quieran involucrarse con este tipo de objetos. Entre las referencias teóricas que invoca se encuentran, por ejemplo, Loss Pequeño Glazier, Eduardo Kac, Katherine Hayles, Chris Funkhouser. 
3 Las definiciones que recorre el texto se proponen establecer distinciones entre los medios que intervienen en las obras, no tanto en relación a sus efectos de sentido o a la dimensión estética de su materialidad. En general, retoman algunas denominaciones más bien por el uso que se les ha dado a los términos y no por un tipo de relación específica con su referente. Por otra parte, se trata de enfoques en los que subyacen reflexiones sobre los tópicos de las teorías de los medios y de la comunicación más que las propias de una preocupación teórica literaria, la que implicaría pensar con más detenimiento de qué manera esas prácticas redefinen las categorías disciplinares, o de la estética, para abonar una consideración profunda sobre la percepción al filo del entre siglo.

Varias de las definiciones que se traen a colación se ocupan del medio "digital" como canal de difusión o como lugar de engendramiento de las obras, pero no profundizan acerca del carácter "literario" que tendría la "literatura" digital (que se presupone en todos los casos, o se lo asocia sin más a la presencia de un componente verbal). Tampoco lo hacen acerca de la instancia de lectura y escritura de modo estricto: las mutaciones de tales experiencias, de las dimensiones témporo-espaciales y de nuestras percepciones sobre ellas parecen no ser tan relevantes a la hora de las definiciones introductorias (no obstante, luego veremos que algunas de estas cuestiones sí se abordan en los artículos compilados).

5 Siguiendo esa línea, el libro se organiza, según anuncia la introducción, en base a las tres etapas que el profesor del Instituto de Tecnología de Georgia (EE UU), Jay David Bolter, ha descripto para los procesos de remediación: el período de transformación y adaptación; el de la literatura digital desde su nacimiento; y el del cibertexto o literatura cyborg (nótese la homologación entre "texto" y "literatura") asistida y producida por máquinas. Si bien la obra enfoca en las realizaciones del género vinculadas a América Latina, en el momento de problematizar los conceptos lo hace mayormente a partir de bibliografías y teorías producidas fuera de América Latina. Esta es una característica significativa de la producción académica al respecto de estos objetos de estudio que, no obstante, sigue una línea constante respecto de lo que frecuentemente ha sucedido en los campos de producción de saber desde los grandes centros.

6 En este mismo sentido, otra cuestión llamativa (al menos desde nuestra perspectiva), es que todas las citas de la introducción, con excepción de las del mexicano Eugenio Tisselli, son citas en inglés: esto nos lleva a suponer que el proceso investigativo que subyace a este texto sigue más bien un movimiento deductivo que inductivo, partiendo de constructos teóricos elaborados para otros corpus (como el de Bolter), que se consideran adaptables al corpus latinoamericano (o viceversa) aun cuando los conceptos que se usen para nombrarlos surjan de corpus distintos, cuando menos, en sus modos de inscripción socio-técnica. El libro nace así del impulso por lograr una especie de muestreo teórico anclado en este tipo de procedimientos, aunque como toda antología o reunión de artículos, deba resignarse a dejar muchos ejemplares afuera de la muestra para poder salir a la luz ${ }^{\text {ii }}$

7 La obra se estructura en tres partes: una primera, denominada "Nuevas formas de expresión poética"; que intenta dar cuenta, desde distintas perspectivas, de diversas facetas de la experimentación literaria con nuevas tecnologías: encontramos allí un abordaje sobre la poesía flash, las videointervenciones (en tanto creadoras de un metalenguaje por el que devienen poesía digital para Angélica Huízar), el problema de 
la traducción expuesto en la poesía como collage multigenérico, la apuesta interactiva de los Clickable poem@s, la robopoética, y la poesía holográfica de Eduardo Kac. Formas de experimentación con nuevos medios que permanecen, en general, invisibilizadas en el campo de lo que se reconoce actualmente como literatura, aunque en el reducido mundo de la literatura experimental con tecnologías se trate, en gran parte, de obras que ya son un clásico (la poesía flash data de los años noventa, mientras que los experimentos holopoéticos nos remontan a 1983).

En el primer apartado, por ejemplo, Osvaldo Cleger conceptualiza la poesía flash a partir de la definición de Katherine Hayles, como una remediación de distintas tradiciones de la poesía visual que comienzan en el caligrama. Propone la programabilidad y los modos de integración de los componentes interactivos y multimodales como rasgos innovadores de esta poesía, a partir del abordaje de Wordtoys (2006) de la argentina Belén Gache, probablemente una de las obras en español de poesía flash sobre la que más se haya escrito.

9 Un rasgo de las obras que se abordan en este libro es la velocidad con la que obsolescen y/o se modifican. Esto supone grandes desafíos a la crítica, que debe seguir el ritmo de tales mutaciones. El caso de lo que Osvaldo Cleger trabaja como poesía flash es uno de estos casos paradigmáticos. En los años noventa y a principios de los dos mil, lo que hoy es Adobe Flash Player era una de las herramientas más versátiles y novedosas para crear contenido interactivo y reproducir video en la web. Actualmente suele ser reemplazada por otras plataformas gráficas que consumen menos recursos de sistema (memoria RAM, baterías) y son menos vulnerables al software malicioso.

Algunos navegadores y sistemas operativos lo bloquearon para impedir que surgieran problemas a causa de sus constantes fallas: Chrome, Firefox, Android, iOS, por ejemplo, restringen su uso. Se ha creado, incluso, un movimiento que incita a que todos los usuarios de internet desinstalen el programa, llamado Occupy Flash ${ }^{\text {iii }}$. Por todo esto, ya no es siempre posible acceder fácilmente a este género de obras en cualquier navegadoriv, y es probable que se vuelvan inaccesibles si no se transponen pronto a otra plataforma que sea integrable y por ello compatible con los estándares industriales que sostienen los navegadores de uso extendido (Ré-Berti, 2017). Algo similar ya ha sucedido con la poesía digital que se almacenaba en disquetes y que actualmente requiere un sistema técnico específico (cuyo uso ya es solo residual) para hacer posible su reproducción y lectura.

10 Por su parte, Eduardo Ledesma aborda la obra TraklTakt del brasileño André Valias, a la que describe como collage intermediático y multigenérico entre poesía, narrativa y ensayo filosófico, en la que se problematiza la traducción de/a nuevos medios en diálogo con distintos conceptos de traducción desde un punto de vista poético, mediático y lingüístico de los poemas de Georg Trakl, a partir de nociones de Ludwig Wittgenstein, de Walter Benjamin, pero también del checo-brasileño Vilém Flusser y de los exponentes del grupo brasileño Noigandres.

11 Scott Weintraub estudia la obra/instalación IP Poetry del argentino Gustavo Romano, a la que ubica en el "género" de "robopoéticas" recurriendo al concepto de Christian Bök, preguntándose por la posibilidad de escribir literatura para robots que no pueda ser comprendida por seres humanos. Así deriva en la pregunta por la autopoiesis en los bots que intervienen en la obra de Romano y sus posibles sistemas cognitivos (por ejemplo, en el aprendizaje de nuevos fonemas), adentrándose en un análisis que apela a ciertos aspectos de la programación, aspectos no siempre considerados en las lecturas 
más habituales de esa obra. Su propuesta sostiene la denuncia de Bök ante el prejuicio antrópico por el cual algunos lectores suelen despreciar las producciones lingüísticas de robots considerándolas "galimatías computarizados". Un juicio que, creo, ha llevado una y otra vez a los críticos a describir los procedimientos involucrados en las obras sin detenerse suficientemente ante todas sus dimensiones con igual atención a sus distintos niveles de complejidadv. Weintraub sostiene que IP Poetry brinda la oportunidad de conocer una conciencia bot embrionaria de primera mano, en el inicio de un proceso de autopoiesis.

12 El texto de Eduardo Acuña-Zumbado presenta y piensa los aspectos novedosos de la Holopoesía del brasileño Eduardo Kac, que data de 1983, así como al sujeto y la subjetividad que presupone este tipo de obra, a partir de una descripción detallada de varias de ellas y un análisis detenido de sus posibilidades de lectura. La problematización de la subjetividad del que produce y lee este tipo de obras también ha sido algo que queda frecuentemente fuera de los textos y lecturas críticas de las obras con nuevos medios. Si bien los primeros holopoemas tienen ya más de 30 años, es menester señalar que no es fácil encontrar, en el mar de artículos que existen sobre estas obras, otros análisis que profundicen en la experiencia de lectura y que se independicen de la descripción general de las obras que hace el propio artista. Para Acuña-Zumbado, la fluctuación constante del signo holopoético configura una especie de lector incómodo, una multiplicidad de subjetividades que se dispersan en las coordenadas espacio-temporales que configura la experiencia de lectura.

La segunda parte del libro, "Nuevas intervenciones poético-políticas"; sugiere una invitación a pensar mayoritariamente obras que explicitan su politicidad. Resulta poco claro, sin embargo, en qué sentido no sería una intervención po/ético-política la denuncia del prejuicio antrópico que comentamos en el apartado anterior, por ejemplo. Cualquiera de los textos del libro podría estar incluido en este apartado, no obstante, se seleccionaron los que reúnen como característica fundamental la tematización de lo político.

El texto de Hilda Chacón aporta observaciones respecto a performances documentadas en la web (ya sea mediante fotos o videos) que la autora presenta como expresiones contestatarias ante la cultura global estadounidense y el capitalismo avanzado, expresiones que se enmarcan en el debate modernidad-posmodernidad tal como se da en Latinoamérica. La autora señala lo que aún no ha hecho el libro explícitamente: es imposible separar el análisis de la literatura de la historia política de América Latina al abordar su producción cultural. Más aun, considerando que la poesía está vinculada, en latinoamérica, a la constitución de los Estados-Nación. No obstante, Chacón busca definir una "poética del horror" fundada en ideas revisitadas de Fredric Jameson y Jesús Martín Barbero, considerando que la frontera móvil entre autor-espectador, característica que encuentra en el ciberespacio, posibilitaría "crear sistemas comunales (...) capaces de incentivar solidaridades y activismos más allá de las fronteras geográficas, culturales e idiomáticas de los lectores-espectadores". El trabajo presenta una visión un tanto inocente de las posibilidades de la red: a la utopía instalada justamente- por el impulso globalizador, referida a la constitución de una comunidad solidaria y sin fronteras viabilizada por la red, le subyace una contracara ominosa en lo que Antoinette Rouvroy llama gobernamentalidad algorítmica, y sobre la cual sería pertinente reflexionar en el marco de la distribución diferencial de la producción de algoritmos, y más aún si la propuesta es pensar la producción cultural latinoamericana. 

papel de la modernidad en las zonas periféricas del capitalismo global. Este segundo apartado presenta también trabajos sobre Las afinidades electivas como red afectiva de líneas que echa luz sobre vinculaciones imprevistas entre poetas; los videopoemas de Anuar Elías desde una perspectiva ética y estética; la Tuiter-poesía generada por Eduardo Navas sirviéndose de la aleatoriedad y la potencialidad de la plataforma Twitter; o las transformaciones de los mecanismos de producción, circulación y recepción de obras cuando interviene la máquina o el software (algo que también aparece en el estudio de Gómez Olivares respecto de la poesía mexicana, o en el de Alamir Aquino Correa sobre la contribución brasileña a la poesía contemporánea, ambos incluidos en la última sección del libro). Llama la atención, sin embargo, que en los trabajos de un apartado así denominado no aparezca con fuerza una reflexión ético política sobre la tecnología y sobre sus efectos en las prácticas situadas. La cita poco atendida de Eugenio Tisselli en la introducción daba pie para incluir también este aspecto en la discusión; aspecto que es uno de los más urgentes para Latinoamérica.

En la tercera parte del libro, "Teorizar nuevas cartografías virtuales", aparece con mayor claridad el panorama regional: allí se ofrecen inventarios, recorridos o textos enciclopédicos (típico recurso en los textos de Belén Gache) que presentan realizaciones del fenómeno en distintas latitudes. Aquí, por ejemplo, el trabajo de Claudia Kozak recupera definiciones de su libro (cfr. próximo apartado) y presenta un minucioso recorrido por la tecnopoesía experimental argentina que parte de los textos radiogénicos de Kin Taniya (Luis Quintanilla), pasando por la poesía Pau Brasil de Oswald de Andrade, Xul Solar, Edgardo Antonio Vigo, hasta llegar a los contemporáneos Mariano Sardón, Leonardo Solaas, Mauro Césari, entre otros.

Lizabel Mónica y Rachel Price exponen un mapa de lo que denominan la incipiente literatura digital cubana y reflexionan sobre sus especificidades, señalando aquellas que la distinguen radicalmente del resto del continente, vinculadas a cuestiones sociales, históricas y políticas de la isla. Su objeto de estudio se configura mayoritariamente por un conjunto de textos que circulan en blogs y twitter, aunque también da cuenta de obras musicales, multimediales, performáticas, digitales.

Luis Correa-Díaz presenta un panorama de lo que llama poesía cibernética latinoamericana desde 1950 hasta 2010. Se trata de lo que todavía está "in print", según el concepto que acuñó para referir a manifestaciones en libros del fenómeno de las "nuevas tecnologías" en el marco de un contexto predigital, pero que se distingue de la pre-computerized poetry descripta por C. Funkhouser. El panorama que Correa-Díaz presenta en este artículo focaliza en obras en las que detecta una "interpenetración de los efectos culturales y estéticos de lo new media o digital en libro". En su corpus, latinoamericano (principal pero no únicamente chileno), que contrasta con obras de otras geografías, esto se da mayoritariamente en el orden de los temas, pero también aparece en la inclusión de pantallas, links en textos "tradicionales", libros con criptogramas o código $\mathrm{QR}$, entre otras alternativas.

Si la poesía es un lugar marginal en los estudios literarios, y la poesía digital lo es aun más, menos generoso suele ser el espacio que se destina a la poesía sonora, muchas veces incomprendida y dejada de lado en nuestra cultura, eminentemente visual. El trabajo de Felipe Cussen parte de la descripción de un poema visual de Augusto de Campos para adentrarse en las distintas resoluciones que suscitaron sus versiones sonoras. El autor incluye el timbre, el tempo, el volumen, el tono, los matices de la voz y 
sus reverberaciones como categorías de análisis. Estos elementos suelen ser despreciados en los estudios de literatura digital que, ante la concurrencia de componentes audio-visuales se enfocan muy frecuentemente en los aspectos visuales, relegando lo sonoro al estatuto secundario de ser apenas ilustración de lo visual. La música es probablemente la primera poética en recurrir explícitamente a instrucciones de uso (en forma de partituras), algo muy frecuente en la literatura digital y nada habitual en la literatura tradicional (salvo excepciones como la de Rayuela), donde las instrucciones de uso se presuponen y son, para cualquier obra, las mismas. Los estudios de poesía sonora como este resultan escasos y valiosos en la búsqueda de desnaturalizar o cuestionar indirectamente las jerarquías sensoriales que rigen en nuestra cultura.

Algunas consideraciones finales

21 Al decir que el libro en cuestión es el primero en preguntarse por las poéticas digitales que se producen en/con/desde América Latina, cabe señalar que existen importantes antecedentes en este ámbito. Para que un trabajo de esta envergadura exista, hubo antes muchos investigadores de diversas latitudes preguntándose por estas obras, publicando de manera menos sistematizada y más dispersa, en general, en revistas o fascículos de visibilidad más restringida. Correa-Díaz señala algunos de estos trabajos en las notas a la introducción. Además de los estudios que miran la producción de diversos países de Latinoamérica desde el otro lado de sus fronteras, hay también una producción de alcance regional y nacional en distintos lugares del continente acerca de la literatura digital, que trabaja en la constitución de un campo crítico sobre y desde nuestros territorios (el ejemplo ineludible en mi país es el de Tecnopoéticas argentinas. Archivo blando de arte y tecnología, editado en 2012 por Claudia Kozak, que es un excelente trabajo de exploración, sistematización y análisis, y el primero por estas tierras en involucrarse explícitamente con las poéticas tecnológicas argentinas).

Retomando algún planteo inicial, uno de los detalles que saltan a la vista en Poesía y poéticas digitales. Electrónicas / tecnos / new-media en América latina: definiciones \& exploraciones es que el libro configura su objeto de estudio y su interés en las producciones de América Latina, pero lo hace, sobre todo, desde América del Norte: sus editores son académicos en Estados Unidos, característica que comparten la mayoría de los autores que presenta el ejemplar; o han pasado por períodos de formación en el hemisferio norte, con alguna notoria excepción. Algo que debemos tener en cuenta al enfrentarnos a esta obra de referencia, entonces, es que si bien muchos de los autores tienen un origen latinoamericano, la mirada analítica sobre el objeto de estudio es mayormente el producto de sus interacciones académicas e indagaciones en espacios socio-técnicos divergentes a la experiencia latinoamericana respecto de la incidencia de las "nuevas tecnologías" en el campo de la literatura y las artes. En este sentido, es sintomático que la única definición citada en la introducción que asume una posición crítica y cuestiona el espacio técnico que da lugar a estas prácticas sea la menos comentada.

23 Siguiendo el planteo, es sumamente interesante reflexionar acerca de las aduanas por las que pasa y en/entre las que se produce el conocimiento sobre estos objetos: la obra de Correa-Díaz y Weintraub habilita amplias reflexiones al respecto. Asimismo, la reflexión que allí se presenta sobre los medios rehabilita problemas de la teoría literaria que estaban solapados en el concepto de libro como soporte: se ha pensado por siglos que la literatura era sólo un contenido distribuido mediante diferentes canales. Si hay algo que ponen en evidencia los experimentos con nuevos medios es que la 
literatura no se agota en su contenido semántico (o que la significación no se agota en el significado de un texto) sino que la materialidad del componente verbal se ve necesariamente implicada en la significación. Estas problematizaciones invitan a revisar nuestra concepción del libro y de nuestras propias prácticas naturalizadas, lo cual es una de las más grandes potencias de la literatura digital: auspiciar una revolución disciplinar respecto incluso de los viejos objetos de estudio, cuyos efectos más contundentes no están tal vez en cómo miramos desde aquí hacia el futuro, si no en cómo volvemos a mirar el pasado.

Esta obra expresa la intención de dar cuenta de la interacción entre poesía y nuevas tecnologías en Latinoamérica mediante la presentación de estudios panorámicos y de casos puntuales, para sacar de una "cierta invisibilidad" (cita a Andrés Burbano) a aquello que se produce en nuestros países. Esa cierta invisibilidad tiene que ver justamente con particularidades históricas y políticas de los canales de circulación y difusión de las prácticas y de los saberes, y con la segregación de estos problemas a espacios marginales en las unidades académicas de aquí y de allá, entre otras. El libro efectivamente logra poner en escena obras que se producen en nuestra región, se publica y se distribuye en forma gratuita, y se lee y se reseña en distintos países, como en este caso.

Sin embargo, la distribución diferencial de lo tecnológico en los distintos territorios del continente, la imposición (o la adopción acrítica) de modelos socio-técnicos generados en el hemisferio norte y la especificidad de los fenómenos intra-técnicos llaman a ser pensados en relación al contexto social, cultural, económico y político en el que las obras se insertan. Claramente la mirada organizadora del libro busca ser hospitalaria, aunque no deja de estar fuertemente situada. Es siempre enriquecedor, no obstante, conocer las múltiples miradas respecto de nuestros objetos. La diversidad de perspectivas ayuda a completar la complejidad de dimensiones del fenómeno que pueden analizarse. Más allá de estos comentarios de corte más bien sociológico, los estudios panorámicos y de casos puntuales que el libro presenta en su interior son, en general, interesantes y sugerentes en sus propuestas.

El resumen de este libro comienza así: "En cualquier expedición, el paso que importa es el primero". El primer paso ha sido dado y se ha iniciado el diálogo: el movimiento es muy promisorio. El tiempo dirá con precisión en qué medida las preocupaciones que aquí recuperan los autores son representativas de lo que se plantea el arte, la literatura y la crítica latinoamericana, y echará luz sobre las inquietudes que no hayan sido abordadas, para dar pie al segundo paso, de entre muchos más, en esta atractiva expedición.

\section{BIBLIOGRAFÍA}

CORREA-DÍAZ, Luis y WEINTRAUB, Scott (Ed.), (2016), Poesía y poéticas digitales / electrónicas / tecnos / new-media en América Latina. Universidad Central, Universidad de Hampshire y Universidad de Georgia. Bogotá, D. C. <http://goo.gl/rILOFz>. 
KOZAK, Claudia (Ed.), (2012), Tecnopoéticas argentinas. Archivo blando de arte y tecnología. Caja Negra, Buenos Aires.

RÉ, Anahí y Berti, Agustín (2017), “Estándar y poéticas industriales en la literatura digital argentina", en dossier especial "Word and Image in Latin American Poetry", en A Contracorriente: revista de historia social y literatura en América Latina, North Carolina State University (en prensa, noviembre de 2017).

\section{NOTAS FINALES}

i. Cybertext: perspectives on ergodic literature (Espen Aarseth, 1997), Digital poetics: the making of epoetries (Loss Pequeño Glazier, 2002), Radiant textuality: literature after the world wide web (Jerome McGann, 2004), Electronic literature: new horizons for the literary (Katherine Hayles, 2008), Beyond the Page: Latin American Poetry from the Calligramme to the Virtual (Angélica Huízar, 2008), New media poetics: contexts, technotexts, and theories (Adelaide Morris y Thomas Swiss, 2009), por ejemplo.

ii. Casi del mismo modo que esta reseña, que no tiene más remedio que limitarse a comentar sólo algunos contenidos de este libro de más de quinientas páginas.

iii. Cfr. http://es.occupyflash.org

iv. En efecto, al ingresar a la primera pieza de la obra, un letrero anuncia: "A partir de su actualización de abril de 2015, Google ha tomado la decisión de bloquear definitivamente un gran número de plugins, entre ellos Java, Adobe Reader o Shockwave, debido a problemas de seguridad de su navegador. Por esa razón, este trabajo incluido en los Wordtoys no podrá verse más en Chrome. RECOMENDAMOS UTILIZAR FIREFOX O CUALQUIER OTRO NAVEGADOR PARA VER ESTA ANTOLOGÍA DE TRABAJOS." (en mayúscula en el original).

v. Como sugerimos más arriba, e incluso en otros textos, centrarse en el procedimiento en detrimento de todos los demás componentes y hablar como si de la totalidad de la obra se hablara es, cuando menos, una lectura miope que se describe perfectamente en la postulación de un prejuicio antrópico.

\section{AUTOR}

\section{ANAHÍ ALEJANDRA RÉ}

Centro de Investigaciones y Estudios sobre Cultura y Sociedad-CONICET-Universidad Nacional de Córdoba, Argentina. 